\title{
Wetting of topographically structured surfaces by crystalline phases
}

\author{
A Esztermann and $\mathrm{H}$ Löwen \\ Institut für Theoretische Physik II, Heinrich-Heine-Universität Düsseldorf, Universitätsstraße 1, \\ 40225 Düsseldorf, Germany \\ E-mail: ansgar@thphy.uni-duesseldorf.de
}

Received 24 November 2004

Published 18 February 2005

Online at stacks.iop.org/JPhysCM/17/S429

\begin{abstract}
The physics of wetting phenomena at structured surfaces by crystalline layers as investigated by theory, computer simulation and experiments is reviewed. Both realizations on the molecular scale and more mesoscopic realizations in colloidal models systems are included. We explore how a crystalline wall pattern affects the wetting by a crystalline phase in the context of a simple hard sphere model relevant for sterically stabilized colloids. We further discuss decoration lattices generated by adsorption of colloidal particles on stripepatterned substrates. For molecular systems, the influence of a rough and preplated surface on triple-point wetting of hydrogen is calculated. Finally, we present data for fluid layering in primitive model simulations of charged colloids near neutral walls.
\end{abstract}

\section{Introduction}

While the traditional wetting transition by a liquid or vapour phase is well understood by now, the same problem is much harder if the 'wetting' phase is a crystal. The reason is that the translational symmetry is broken in the crystalline phase and thereby much more complex wetting scenarios evolve such as discrete crystal layering [1, 2]. Moreover, in contrast to a fluid, a solid can carry elastic shear stress which provides new physics in the wetting by crystalline phases. In particular, we expect a wealth of novel phenomena if the wall which is wetted carries a structural (e.g. periodic) pattern which favours certain crystalline structures. This is an active research field and we shall review some results obtained in the past few years in this paper.

However, three other (and simpler) cases are much better understood, namely:

(i) structureless (i.e. smooth) walls;

(ii) a periodic pattern in the bulk;

(iii) laterally structured walls in an inhomogeneous liquid far away from a wetting transition. 
All these cases are necessary prerequisites for the wetting of a structured wall by solid phases. Therefore, let us mention some basic work in these subtopics first.

In the case of a wall without lateral structure, the wetting behaviour of a crystalline phase has been intensely studied for a simple hard sphere model close to a hard wall. Based on computer simulation studies [3,4] and density functional theory [5], it was shown that there is a precrystallization transition. This implies that there is a molecularly thick wetting layer of several crystalline layers close to bulk fluid-solid coexistence when the stable bulk phase is fluid. Details of this precrystallization transition, however, are still unclear. Recently, the model has been used to study heterogeneous crystal nucleation close to a smooth wall [6].

Periodic external fields in the bulk lead to novel bulk phase diagrams with re-entrant effects; for recent reviews see [7,8]. This was demonstrated in experiments with colloidal suspensions in crossed laser beams [9, 10]. If the resulting external field is compatible with a crystal structure, then a stable crystal is obtained even if the crystallization transition is far away in the field-free case. Such a laser-induced freezing was predicted by computer simulations of microscopic models [11], by density functional calculations [12], and by Landau-type theories [13].

Finally, for case (iii), the influence of a topographical wall structure-as modelled by wall particles fixed on a triangular lattice — on structure and shear behaviour was investigated by computer simulation [14]. Such a wall structure will greatly influence crystalline layering transitions for small plate distances [15-17]. Studies with chemically heterogeneous patterns (e.g. periodic stripes) are much more popular; see e.g. [18-22] and the papers by Lipowsky, Dietrich, Krausch, Herminghaus, Rühe in this special issue. The aim of the paper is twofold: first, we intend to review recent progress in the field of wetting of structured substrates by a crystalline phase both for molecular and colloidal model systems. We discuss step by step the case of a periodic wall pattern offered to a bulk hard sphere fluid where we review recent results obtained by theory, computer simulation and real-space experiments on sterically stabilized colloidal suspensions. We further address the formation of colloidal monolayer decoration lattices on periodic substrate structures. Then we focus on triple-point wetting of molecular systems such as hydrogen on different substrates where the roughness turns out to play a crucial role. The second goal of the paper is to present some original results: first, a theory for triple-point wetting in the case of a preplated substrate is presented which generalizes the theory of previous approaches. Here we discuss possible experimental implications.

The wall structure is classified by two extremes: in the first case, the characteristic length scale of the wall pattern is comparable with the interparticle spacing of the bulk fluid. This is a situation frequently encountered for colloidal systems where the interparticle spacing is of the order of micrometres. The surface structure can then be realized either by chemical etching or other lithographic techniques [23, 24] or by fixed colloidal spheres on a substrate [25-34]. Even a periodic wall pattern by many fixed colloidal spheres can be realized. Examples are discussed in section 2. In the second limit, the wall structure varies on a length scale much larger than a typical interparticle spacing. This limit is encountered for wetting by molecular systems on rough substrates, such as hydrogen on gold. This case is extensively discussed in section 3 . We further present computer simulation results for density profiles of charged colloids near a hard wall modelled within the primitive model with explicit counterions. Finally, we address the case of a smooth but charged wall in the context of charged colloidal suspensions in section 4 .

\section{Wetting of walls which are patterned on a 'molecular' scale}

If sub-micrometre wall patterns are exposed to a bulk colloidal suspension, there is a competition between bulk interparticle correlations and the length scale of the wall pattern. 
This can give rise to various novel self-organization effects at the wall. In this chapter we review in particular wetting of periodically structured walls by colloids.

\subsection{Wetting of periodically structured hard walls by hard spheres}

The wetting behaviour of periodically structured walls by a hard sphere fluid was recently studied extensively by theory, computer simulation [35-37] and real-space experiments [38]. When fixed hard spheres form a periodic lateral array on the wall [39-43], one may ask whether the system prefreezes by picking up the two-dimensional lattice symmetry.

In the simulations, different substrate patterns were investigated: triangular lattices, square lattices, zig-zag stripe and rhombic patterns. The first three patterns arise if an fcc crystal is cut along the (111), (100), and (110) orientations. The rhombic pattern, on the other hand, does not correspond to a thermodynamically stable crystal but rather to a sheared fcc crystal. In the first case, the mismatch between the lattice constants of the wall crystal and the coexisting bulk crystal is the essential parameter: a necessary condition for complete wetting by the undistorted bulk crystal is that the two lattices fit perfectly. The simulation data [35-37] show that even for perfectly matching lattice constants, there is complete wetting only for the (111) pattern but not for the (110) and (100) patterns. Furthermore, the formation of crystalline layers happens in a discontinuous way via a layer-by-layer growth mode. A first crystalline layer is formed well below the bulk crystallization transition. For a rhombic pattern, there are two possibilities; either there is incomplete wetting by a few layers, or there is no wetting at all. For a triangular pattern, the freezing of the first layer can be enhanced if the lattice constant is larger than that of the coexisting bulk crystal.

Via phenomenological approaches which combine thermodynamics, elasticity theory and effective interface potentials, scaling relations were predicted $[35,36]$ which were confirmed by the simulations. Real-space studies [38], on the other hand, have confirmed the first-order transition in the first layer. Further studies on different periodically structured templates made by fixed hard spheres exactly realize the hard sphere simulation model. The behaviour predicted in the simulation was basically confirmed for different surface orientations [44]. Finally, the layer-by-layer growth mode was also found in binary crystals [45].

There is still a debate about complete wetting of a structureless (smooth) hard wall. The wall-fluid, wall-solid and fluid-solid interfacial tensions are known by computer simulation [46-48] but the statistical errors are too large to discriminate complete wetting from incomplete wetting. A recent direct computer simulation [4] gives evidence for the fact that there is complete wetting but it starts close to bulk coexistence.

Finally, let us mention a complementary case of a wall structure which disfavours crystalline layers. Combined with a strong attractive interaction one can then achieve local high densities at the wall while the structure remains fluid-like. In a recent simulation study [49] it was investigated whether the wall induces a fivefold symmetry in the liquid which was found in recent scattering data [50]. The simulation data of [49] revealed that the degree of fivefold symmetry is governed more by the local density than by the wall itself. The wall suppresses the fivefold symmetry only in the first two layers adjacent to the wall. This confirms the inherent assumption made in the interpretation of recent scattering data [50] that the surface-sensitive experiments basically probe the degree of fivefold symmetry in the bulk.

\subsection{Colloidal decoration lattices on stripe-patterned substrates}

An interesting competition arises when colloids are adsorbed on attractive stripe-patterned walls. The widths of the stripes and the interstripe distance then provide further length scales 
to the hard core of the colloidal particles. From a competition of these length scales, it was shown [51] that a wealth of different stable decoration lattices occurs such as triangular, quadratic, rhombic, kite-like and sheared honeycomb lattices and triangular slices as well as triangle superlattices. This theoretical result is of relevance for growing crystals of unusual structure: the decorated substrate may be offered as a template to other mobile colloidal particles in order to nucleate further colloidal crystalline sheets and to grow 'exotic' colloidal bulk crystals $[39,52]$. One may speculate that growing a metastable quasicrystal would be possible if a suitable template structure is taken.

\section{Wetting of rough substrates by crystalline sheets}

The problems dealt with in the previous chapter can be classified as rough substrates where the typical variation of the roughness is in the microscopic scale, i.e. of the order of the interparticle spacing. We now focus on the opposite case where the roughness is characterized by a length scale much larger than the interparticle distance as relevant for wetting of substrates by molecular solids (such as e.g. hydrogen films). For idealized completely flat substrates, a thorough theoretical understanding of wetting has been achieved by now and it is possible to predict the thickness of a liquid film as a function of the substrate-particle and interparticle interactions for given thermodynamic parameters such as temperature and pressure. However, if the temperature $T$ of the system is below the triple temperature $T_{3}$, a solid film can form on the substrate near the sublimation line. The authors of [53-56] have shown in their experiments that a solid film wets a solid substrate only incompletely, that is, the width of the solid film remains finite when approaching gas-solid coexistence.

The solid film experiences elastic compression induced by the substrate attraction which it cannot relax. These strains lead to incomplete wetting [35]. This fact is also the basis of the traditional Gittes-Schick theory [57] of solid adsorption on flat substrates. However, the Gittes-Schick theory predicts that, for a particular value $R_{0}$ of the Hamaker constant $R$ of the substrate attraction, complete wetting by a solid layer is possible. Furthermore, for $R>R_{0}$, the thickness of the solid film $\ell_{\mathrm{s}}$ decreases with increasing $R$. This is in contrast to liquid wetting. The Gittes-Schick theory deals with wetting on flat substrates only. However, real substrates used in experiments will always exhibit a certain roughness. In the following we will focus on the role of this substrate roughness and describe a simple theory which proves that surface roughness plays a key role in wetting by a solid. Even a small roughness inevitably leads to incomplete wetting and our theory predicts, for realistic parameters, a considerable reduction in the film thickness $\ell_{\mathrm{s}}$ compared to the Gittes-Schick theory. Complete wetting by a solid no longer occurs for any choice of $R$. Therefore, we can show that the substrate roughness plays a decisive role in triple-point wetting and the assumption of a flat surface is not justified. Parts of this theory have already been published elsewhere [58] where the predictions were compared successfully to experimental data for hydrogen on gold substrates; here, we describe more details and also include the preplated case.

Holding the system at a fixed temperature $T$ and pressure $P$, we calculate the excess grand potential $\Sigma$ per unit area with respect to a non-wetting situation. This can be done conveniently by using density functional theory in the local density approximation with meanfield corrections [59].

We model the system as follows: the substrate is taken to lie in the $x y$-plane at $z=0$, but modulated by a roughness function $\zeta(x, y)$ which is supposed to vary on a typical length scale $h$ in the vertical and $b$ in the lateral direction where $b \gg h, \sigma$, with $\sigma$ being a molecular length scale. The substrate is preplated by a solid sheet of thickness $d$ which has another Hamaker constant $H^{\prime}$ than that of the bulk material which we denote by $H$. By varying the preplating 


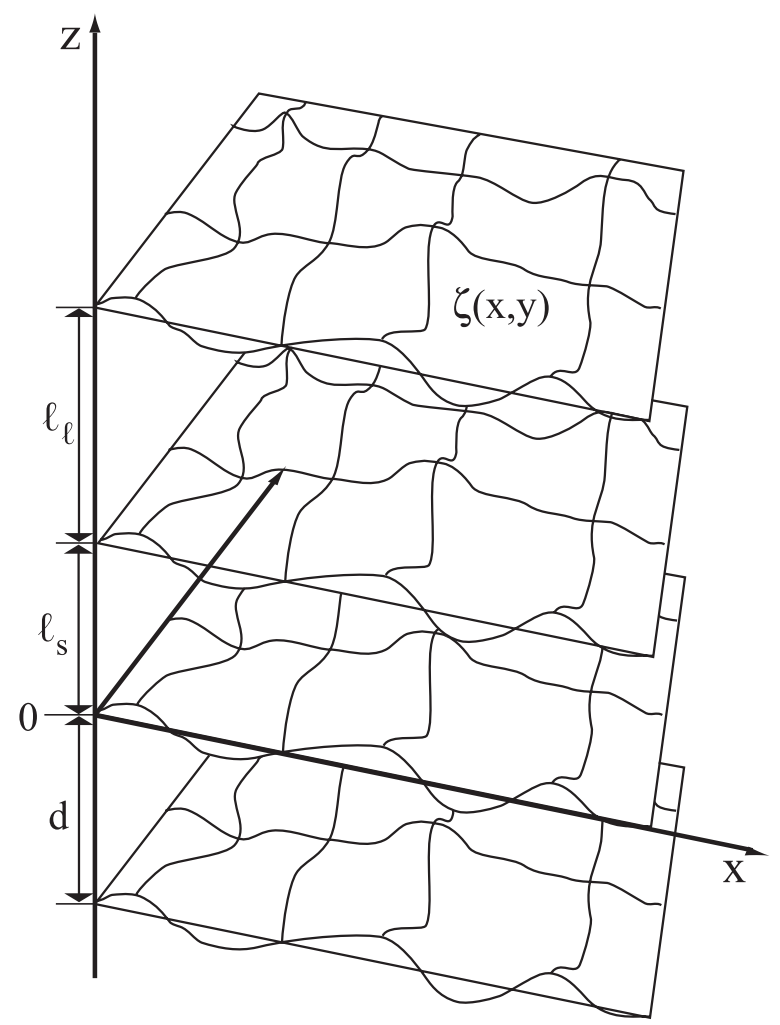

Figure 1. Situation of a preplated substrate. The preplate has a thickness $d$; the solid and liquid wetting layers possess a thickness $\ell_{\mathrm{s}}$ and $\ell_{1}$. The substrate roughness is described by a height function $\zeta(x, y)$.

thickness one can tune the effective Hamaker constant in the range between $H^{\prime}$ (for large $d$ ) and $H$ (for small $d$ ). The whole situation is illustrated in figure 1. The preplated substrate is covered by a solid slab of thickness $\ell_{\mathrm{s}}$. On top of the solid lies a liquid slab of thickness $\ell_{1}$.

The excess grand potential $\Sigma$ is the sum of three terms, $\Sigma=\Sigma_{1}+\Sigma_{2}+\Sigma_{3}$, which represent thermodynamic free energies, the lateral elastic strain in the solid and the elastic free energy cost of a distorted solid picking up the substrate roughness. The thermodynamic part involves the Hamaker constants which give rise to van der Waals attraction. In the traditional approach of the sharp-kink approximation of a mean-field density functional [59], the full density field is approximated by a stepwise constant ('sharp-kink') function as shown in figure 2.

We shall follow this approach and generalize it towards a situation of a preplated substrate. The resulting thermodynamic part of the excess grand potential per unit area, $\Sigma_{1}$, is then given by

$$
\begin{aligned}
\Sigma\left(\ell_{\mathrm{s}}, \ell_{1}\right)=\gamma_{\mathrm{ws}} & -\gamma_{\mathrm{wg}}+\gamma_{\mathrm{s} l}+\gamma_{\mathrm{lg}}+\ell_{\mathrm{s}} \Delta p_{\mathrm{s}} \frac{\rho_{\mathrm{s}}}{\rho_{\mathrm{g}}^{\mathrm{s}}}+\ell_{1} \Delta p_{1} \frac{\rho_{l}}{\rho_{\mathrm{g}}^{\ell}} \\
& +\frac{\left(\rho_{\mathrm{s}}-\rho_{l}\right) C_{\mathrm{p}}}{2 \ell_{\mathrm{s}}^{2}}+\frac{\left(\rho_{\mathrm{s}}-\rho_{l}\right)\left(C_{\mathrm{s}}-C_{\mathrm{p}}\right)}{2\left(\ell_{\mathrm{s}}+d\right)^{2}}+\frac{\rho_{l} C_{\mathrm{p}}}{2\left(\ell_{\mathrm{s}}+\ell_{1}\right)^{2}}+\frac{\rho_{l}\left(C_{\mathrm{s}}-C_{\mathrm{p}}\right)}{2\left(\ell_{\mathrm{s}}+\ell_{1}+d\right)^{2}} \\
& -\left(\rho_{\mathrm{s}}^{2}-\rho_{\mathrm{s}} \rho_{l}\right) \frac{H}{\ell_{\mathrm{s}}^{2}}-\left(\rho_{l}^{2}-\rho_{\mathrm{s}} \rho_{l}\right) \frac{H}{\ell_{1}^{2}}-\rho_{\mathrm{s}} \rho_{l} \frac{H}{\left(\ell_{\mathrm{s}}+\ell_{1}\right)^{2}}
\end{aligned}
$$

for large $\ell_{\mathrm{s}}$ and $\ell_{1}$ and close to the triple point. 


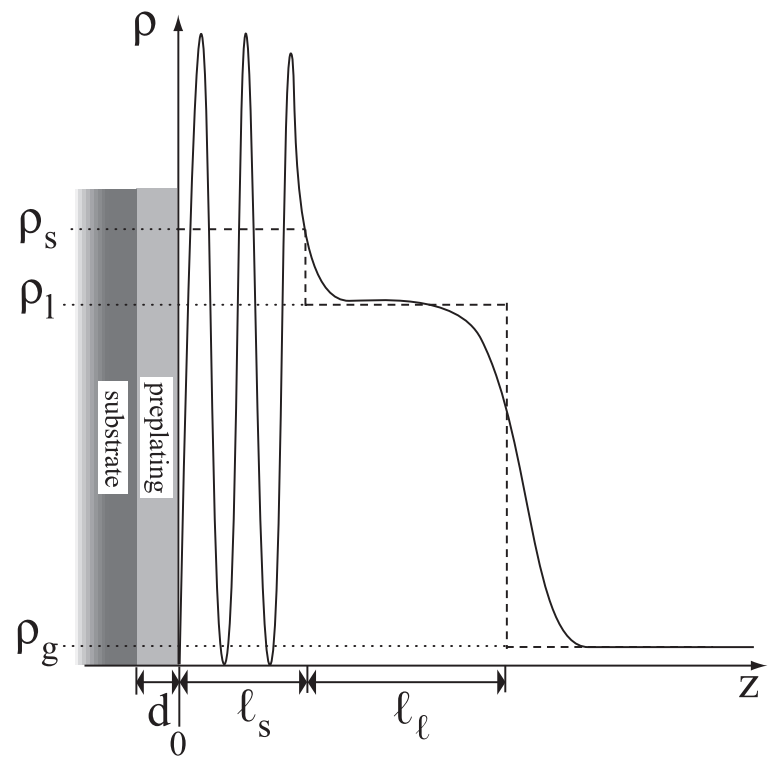

Figure 2. Sharp-kink approximation (dashed) and physical density profile (solid curve).

Here, the quantities $\gamma_{i j}$ are the extrapolated interfacial tensions between wall $(i=\mathrm{w})$, solid $(i, j=\mathrm{s})$, liquid $(i, j=1)$ and gas $(j=\mathrm{g})$, and $P_{0}\left(P_{0}^{\prime}\right)$ is the sublimation (condensation) pressure. The liquid number density at liquid-gas coexistence is denoted by $\rho_{\mathrm{l}}$, while $\rho_{\mathrm{S}}$ and $\rho_{\mathrm{g}}$ are the number densities of solid and gas, respectively, at the sublimation line. We assume $\rho_{\mathrm{g}} \ll \rho_{\mathrm{l}}<\rho_{\mathrm{s}}$. In those regions where the coexistence is metastable, we extrapolate these quantities from their thermodynamically stable regions. The van der Waals tails of the interaction potentials of the adsorbate-substrate $(i=\mathrm{s})$ and adsorbate-preplate $(i=\mathrm{p})$ decay as $-2 C_{i} / z^{3}$, that of the adsorbate-adsorbate interaction as $-12 H / \pi r^{6}$ for large separations $z$ or $r$ where $C_{i}$ and $H$ are the respective Hamaker constants [2].

For the special case of no preplating $(d=0)$ this formula reduces to the well known $[2,58,60]$ result

$$
\begin{aligned}
\Sigma_{1}\left(\ell_{\mathrm{s}}, \ell_{1}\right)= & \gamma_{\mathrm{ws}}+\gamma_{\mathrm{s} 1}+\gamma_{\mathrm{lg}}-\gamma_{\mathrm{wg}}+\frac{\rho_{\mathrm{s}}}{\rho_{\mathrm{g}}}\left(P_{0}-P\right) \ell_{\mathrm{s}}+\frac{\rho_{\mathrm{l}}}{\rho_{\mathrm{g}}}\left(P_{0}^{\prime}-P\right) \ell_{1} \\
& +\frac{A_{1}}{\ell_{\mathrm{s}}^{2}}+\frac{A_{2}}{\ell_{1}^{2}}+\frac{A_{3}}{\left(\ell_{\mathrm{s}}+\ell_{1}\right)^{2}}
\end{aligned}
$$

for large $\ell_{\mathrm{s}}$ and $\ell_{1}$ and close to the triple point. Here, we have further used the following abbreviations: $A_{1}=\left(\rho_{\mathrm{s}}-\rho_{\mathrm{l}}\right)\left(C_{\mathrm{s}}-\rho_{\mathrm{s}} H\right), A_{2}=\left(\rho_{\mathrm{s}}-\rho_{\mathrm{l}}\right) \rho_{\mathrm{l}} H$, and $A_{3}=\rho_{\mathrm{l}}\left(C_{\mathrm{s}}-\rho_{\mathrm{s}} H\right)$.

For large preplating thicknesses $\left(d \gg \ell_{\mathrm{s}}, \ell_{1}\right),(1)$ also reduces to (2), but with $C_{\mathrm{p}}$ substituted for $C_{\mathrm{s}}$.

We note that by a priori setting $\ell_{1}=0$, the theory can also be used close to the sublimation line but away from the triple point.

The next term, $\Sigma_{2}$, describes the free energy of the solid strain caused by the substrate attraction. Its explicit form for large $\ell_{\mathrm{s}}$ was elaborated by Gittes and Schick [57] as

$$
\Sigma_{2}\left(\ell_{\mathrm{s}}\right)=-B S^{2} / \ell_{\mathrm{s}}-2 B S^{3} / \ell_{\mathrm{s}}^{2},
$$

where $B=\frac{3 E}{2(1+v)}, E$ is Young's modulus of the adsorbed solid, and $v$ is its Poisson ratio. The total reduced stress is $S=0.0229\left(R-R_{0}\right) \sigma$ where $R=C / H \rho_{\mathrm{s}}$ is the ratio between 
the adsorbate-substrate and adsorbate-adsorbate interactions. Combining $\Sigma_{1}$ for $d=0$ and $\Sigma_{2}$ yields the Gittes-Schick model. In this model, complete wetting is possible only for $R \equiv R_{0}=1.88$ where $S$ vanishes.

A surface roughness induces an additional bending of the adjacent crystalline sheet. A weakly bent crystalline layer of width $\ell_{\mathrm{s}}$ has the following elastic free energy per area $A$ [61]:

$$
\Sigma_{3}\left(\ell_{\mathrm{s}}\right)=\frac{E \bar{G}}{24\left(1-v^{2}\right)} \ell_{\mathrm{s}}^{3}
$$

with the mean curvature $\bar{G}$ measuring the strength of roughness

$\bar{G}=\frac{1}{A} \iint_{A}\left\{\left(\frac{\partial^{2} \zeta}{\partial x^{2}}+\frac{\partial^{2} \zeta}{\partial y^{2}}\right)^{2}+2(1-v)\left[\left(\frac{\partial^{2} \zeta}{\partial x \partial y}\right)^{2}-\frac{\partial^{2} \zeta}{\partial x^{2}} \frac{\partial^{2} \zeta}{\partial y^{2}}\right]\right\} \mathrm{d} x \mathrm{~d} y$.

It is possible to calculate $\bar{G}$ from experimental data (which are obtained e.g. from atomic force microscopy). Such data, however, typically cover only a small part of the substrate. Therefore, a model surface depending on only a few parameters is desirable. One model is given by sinoidal protrusions on a square lattice

$$
\zeta(x, y)=\frac{h}{2} \sin \frac{2 \pi x}{b} \cos \frac{2 \pi y}{b}
$$

with parameters $h, b$ as introduced earlier. Choosing a rectangular area of integration, $A$, we obtain

$$
\bar{G}=\frac{4 \pi^{4} h^{2}}{b^{4}}
$$

Alternatively, one can put the protrusions on a hexagonal lattice, which yields

$$
\zeta(x, y)=\frac{h}{2} \sin \frac{2 \pi x}{b} \sin \frac{\pi(x+\sqrt{3} y)}{b} \sin \frac{\pi(x-\sqrt{3} y)}{b} .
$$

Taking the area of integration to be a parallelogram with equal sides and an internal angle of $60^{\circ}$, we obtain in this case

$$
\bar{G}=\frac{24 \pi^{4} h^{2}}{b^{4}}
$$

The equilibrium solid and liquid thicknesses are obtained by minimizing $\Sigma\left(\ell_{\mathrm{s}}, \ell_{1}\right)$ with respect to both $\ell_{\mathrm{s}}$ and $\ell_{1}$. The dependence of the wetting layer on the parameters $h$ and $b$ is shown in figure 3 . For $d=0$, a substantial increase of the crystalline layer thickness is only achieved for $h<\sigma$, or for very large $b$.

Contrary to the Gittes-Schick theory, the bending free energy prevents complete wetting by a solid sheet even when the total reduced stress $S$ vanishes since the third term in $\Sigma$ (4) diverges as $\ell_{\mathrm{s}}^{3}$.

In figure 4 it is demonstrated that incomplete wetting occurs for $T<T_{3}$ (with $T_{3}$ denoting the triple-point temperature) where the thickness $\ell=\ell_{\ell}+\ell_{\mathrm{s}}$ reaches only a value of $4 \sigma$ at $P_{0}$ instead of approximately $50 \sigma$ computed for vanishing roughness. Furthermore, the thickness of the wetting layer has been measured experimentally for $\mathrm{H}_{2}$ on Au. Quantitative comparison to these experiments for the unpreplated case $(d=0)$ was published in [58]. The theory was capable of describing the whole set of experimental data.

The dependence of the complete (liquid and solid) wetting layer upon the thickness of a preplate is shown in figure 5. For the Hamaker constant of the preplate, two values have been taken to illustrate the general behaviour. As an example of a strongly attractive preplate, $C_{\mathrm{p}}=2 C_{\mathrm{s}}$ was chosen, while $C_{\mathrm{p}}=0.5 C_{\mathrm{s}}$ represents weakly attractive preplates. In both cases, the thickness of the wetting layer is only slightly changed. 


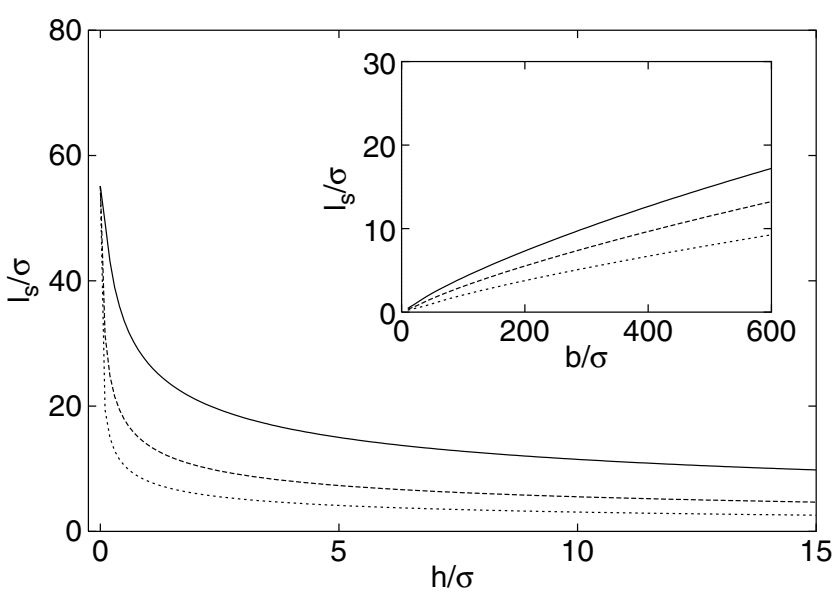

Figure 3. Thickness of a solid wetting layer at the sublimation line in reduced units as a function of valley to peak height $h$ of the roughness profile. A Lennard-Jones model is used with [62] $T=0.585, C=8.7 \varepsilon \sigma^{3}, E=41.7 \varepsilon \sigma^{-3}, v=0.35, \rho_{\mathrm{s}}=0.988 \sigma^{-3}$, and $\rho_{\mathrm{g}}=2.57 \times 10^{-4} \sigma^{-3}$. The different curves are for different lateral roughness parameters $b=100,200,500 \sigma$ (bottom to top). Inset: thickness of the solid layer as a function of the lateral roughness parameter, $b$. Different curves are for different vertical parameters $h=5,10,25 \sigma$ (top to bottom).

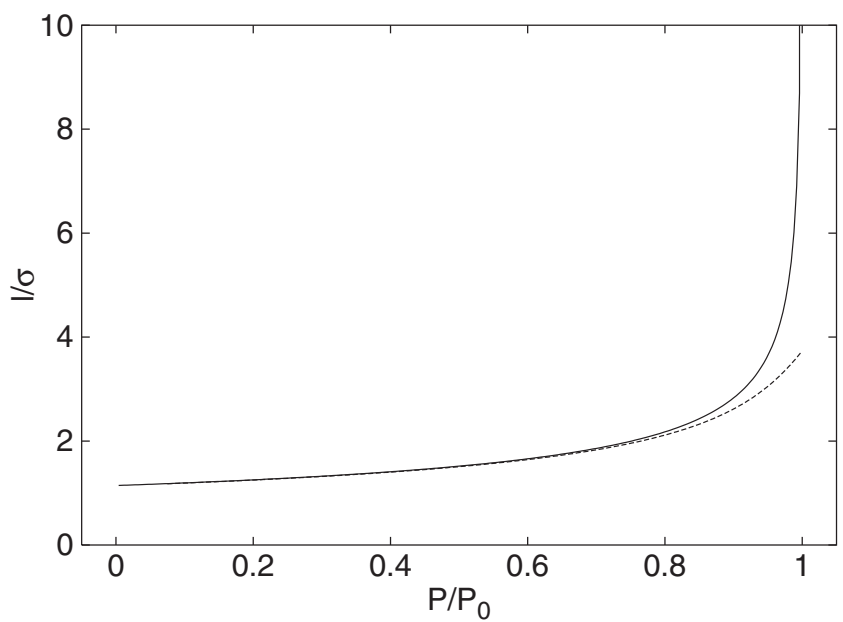

Figure 4. Adsorption isotherm slightly below the triple point. The same parameters as in figure 3 are used. The total wetting layer thickness is shown as a function of pressure in units of the sublimation pressure. The upper curve is for a vanishing substrate roughness $\bar{G}=0$, while the lower one is for $\bar{G}=10^{-3} \sigma^{-2}$. There is a substantial reduction of the wetting layer thickness due to surface roughness near the sublimation line.

Thus, a preplated situation just shifts the thickness of the wetting layer towards a value that would be achieved with a different Hamaker constant (namely, that of the preplate). This shift takes place in a continuous and monotonic fashion; therefore, the fundamental behaviour of the system remains unchanged.

In conclusion, for a smooth wall, the thickness of the solid wetting layer, $\ell_{\mathrm{s}}$, is much larger than for a rough substrate. Even a small finite roughness dictates the width of the crystalline layer. The effect of the roughness is much stronger than strain caused by the substrate attraction. 


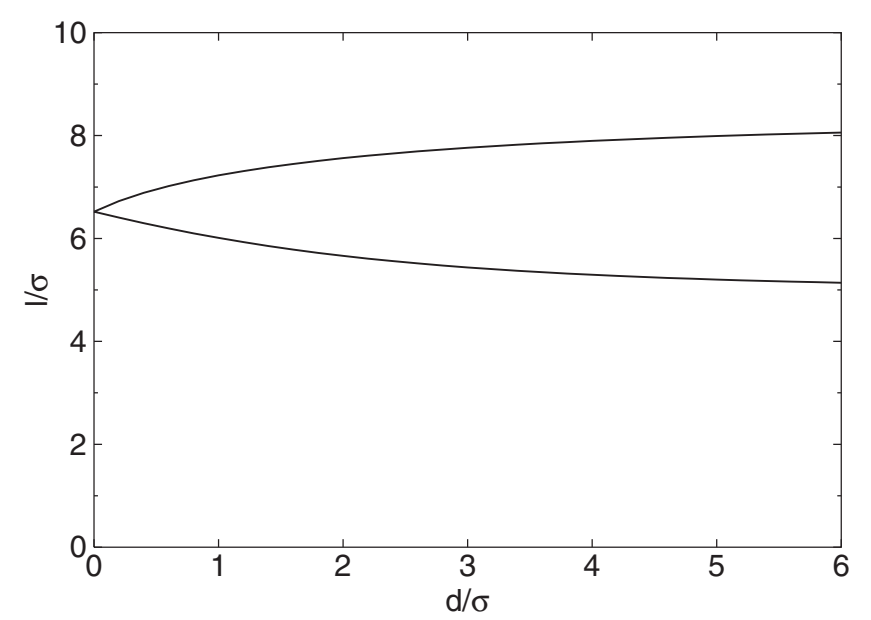

Figure 5. Total thickness of the wetting layer as a function of preplating thickness. Upper curve, $C_{\mathrm{p}}=2 C_{\mathrm{s}}$; lower curve, $C_{\mathrm{p}}=0.5 C_{\mathrm{s}}$. The roughness parameters are $b=100 \sigma$ and $h=15 \sigma$. All other parameters are as in figure 3 .

We have calculated the dependence of the wetting layer thickness on substrate roughness and have shown that a finite roughness always enforces incomplete wetting by a solid adsorbate.

\section{Charged colloids near walls}

While the considerations in section 2 were for neutral colloidal particles, it is tempting to ask whether the charge on the colloidal spheres will alter the wetting behaviour significantly. Dispersions of charged colloids were used in real-space experiments to form crystalline templates [63]. It is fair to say that the theoretical understanding of wetting by charged colloids is much less advanced than that of neutral hard-sphere-like colloids. If one focuses on a simple Yukawa description of the interaction between the colloids which is valid for high salt concentrations, the structure of crystalline layers confined between two parallel walls has been investigated recently, and re-entrant transitions in crystalline bilayers were discovered [17].

However, for deionized suspensions where the Yukawa interaction is expected to break down, much less is known. Near smooth walls, even the theoretical understanding of the effective interaction between charged colloids is far from being complete, but computer simulations have revealed that there are no attractions for monovalent counterions [64]. The simulations are typically done within the so-called 'primitive' model of strongly asymmetric electrolytes with explicit counter- and coions [65]. Simulations of the 'primitive' model for many colloids were mainly done in the bulk $[65,66]$. One more recent study [67] has addressed macroions near a charged wall where the charge asymmetry $Z / q$ between the macroion charge $Z$ and the counterion charge $q$ was limited to 20. Clearly more data are needed for colloidal density profiles near the walls. In particular, if one wants to get insight into precrystallization or wetting by a crystalline sheet one has to simulate close to the bulk fluid-solid coexistence. This problem is much harder to solve than for the hard-sphere fluid since the bulk freezing transition is not yet known and the simulation is more time consuming due to the presence of the additional counterions.

In this section, we present some results of extensive computer simulations for density profiles of charged colloids at or between smooth neutral walls. In figure 6, density profiles for 


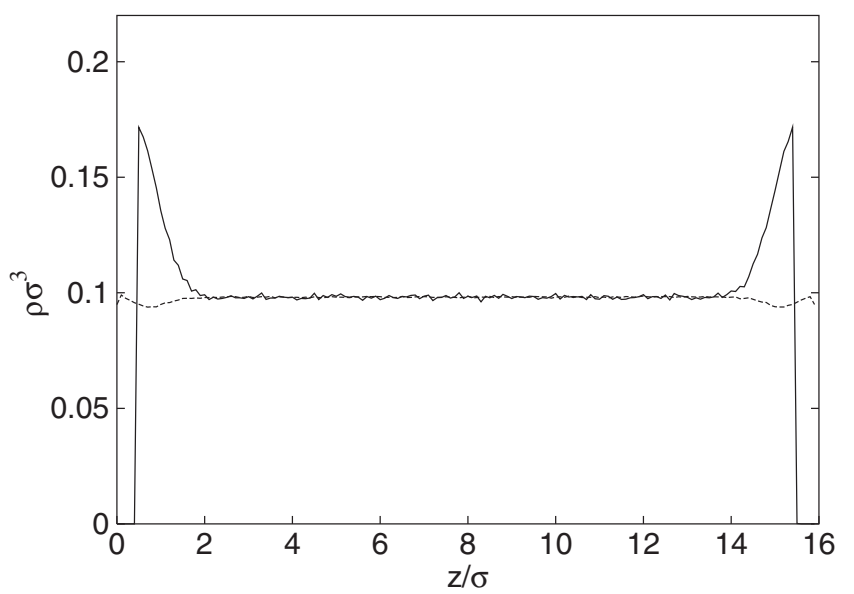

Figure 6. Density profile of a one-component colloidal suspension between hard walls. Solid curve: macro-ions, hard-core diameter $\sigma$, charge $20 e$. Dashed curve: monovalent counterions, hard-core diameter $\sigma / 128$, charge $-e$; the size of the simulation box is $8 \times 8 \times 16 \sigma^{3}$, the Bjerrum length is $\sigma / 128$, and the macro-ion density is $0.098 \sigma^{-3}$. The counterion density has been scaled by a factor of 20 . There is no added salt.

macroions (solid curve) and counterions (dashed curve) are shown for an asymmetry $Z / q=20$. Here, monovalent counterions were considered and no salt was added. The wall separation is $L=16 \sigma$ where $\sigma$ is the hard-core diameter of the colloids which serves as a convenient length scale. The Bjerrum length $\lambda_{\mathrm{B}}=q^{2} / \epsilon k_{\mathrm{B}} T$ is fixed to be $\sigma / 128$ where $\epsilon$ is the dielectric constant of the solvent. The counterions are point particles. The bulk macroion density is $\rho=0.098 / \sigma^{3}$. All parameter combinations will result in a colloidal fluid well away from the bulk freezing transition.

The density profiles exhibit a strong peak at contact with the wall and then decay and oscillate towards the bulk value in the middle between the plates. The fluctuations in the maroion profiles are from statistical uncertainties of the simulation. Since the counterion density is magnified by a factor of $Z / q$ a local coalescence of macro- and counterion densities implies local charge neutrality. Close to the wall, local charge neutrality is strongly violated. This is due to the fact that counterions can get closer to the wall because they are point particles and entropy will force them to spread over the whole space available. This builds up a local electric field at the wall like a little capacitor obtained via colloidal layering. Interestingly, in the layered region macroions and counterion density are alternating; the former is minimal when the latter is maximal.

A higher charge asymmetry of $Z / q=50$ is considered in figure 7 . This results in a larger repulsion between macroions and hence in a larger contact value of the macroion density at the wall. Finally, in figure 8 , the bulk density of the macroion is increased with respect to that used in figure 6 . This means - by global charge neutrality-also a higher counterion density. Again this results in a higher density at contact and more pronounced layering.

Other recent studies have considered charged suspensions near a wall together with a gravitational field where entropic charge lifting for one-component [68-72] and a colloidal brazil nut effect for two-component suspensions [73] was found. The latter implies that the heavier colloidal particles are on top of the lighter ones provided their mass per charge is smaller than that of the lighter ones. The full wetting problem with crystalline layers at the wall has not yet been tackled by simulation and is a challenging problem for future work. 


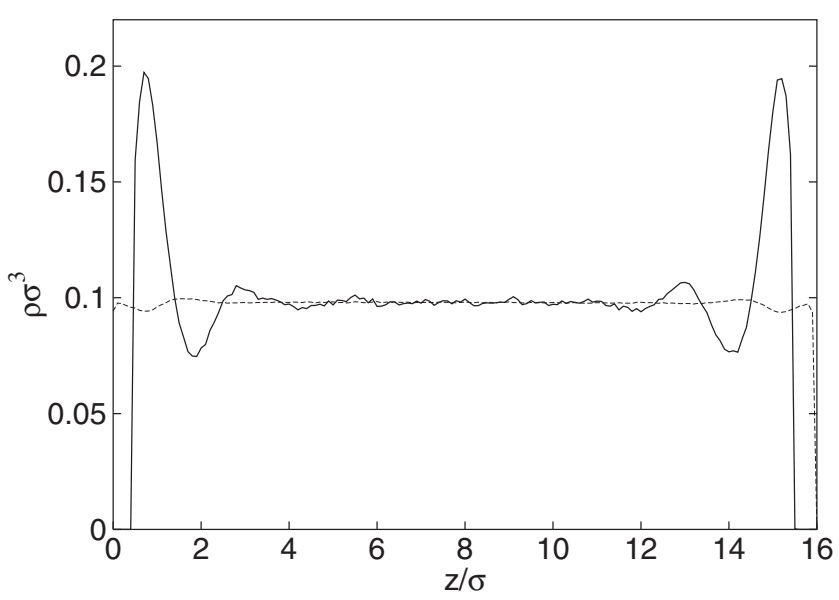

Figure 7. Density profile of a one-component colloidal suspension with a higher charge asymmetry of 50 . The counterion density has been scaled by a factor of 50 . The other parameters are as in figure 6 .

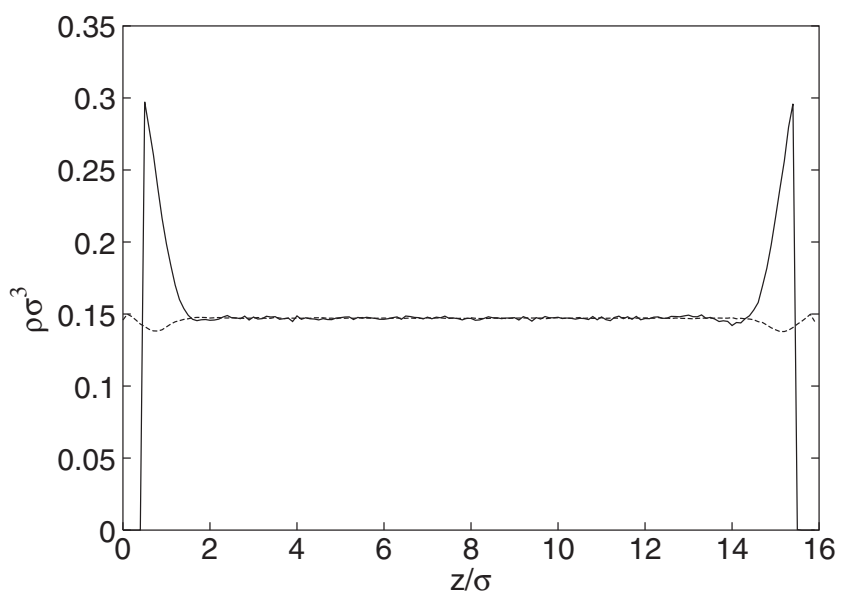

Figure 8. Density profile of a one-component colloidal suspension at a higher density. The macro-ion density is $0.146 \sigma^{-3}$; the other parameters are as in figure 6 .

\section{Conclusions}

If a structured substrate is wetted by a crystalline sheet, the competition between the length scales of the substrate structure and the thermodynamically stable bulk solid gives rise to a variety of different effects: template-induced prefreezing if the substrate structure is favourable for wetting and template-disallowed wetting if the surface structure is incompatible with the wetting solid. Furthermore, new structures can form such as sheared honeycomb decoration lattices on stripe-patterned substrates. Moreover, substrate roughness will strongly influence triple-point wetting by punishing crystalline layers with a bending free energy cost. The interaction of charged colloids is even more subtle and when exposed to a charged patterned substrate more wetting effects are conceivable which still have to be discovered in detail.

In all parts of this paper, we have neglected the role of crystal defects in solid layers which can lower the free energy cost of the elastic strain. This should be an important point of future 
work. The resulting structures play a key role to stimulate crystal growth of exotic lattices needed e.g. for the construction of optical bandgap materials. The resulting wetting structures may further be useful to fabricate micro- and nanofluidic devices.

\section{Acknowledgments}

We thank H M Harreis, M Heni, J Klier, P Leiderer, M Sohaili, and A van Blaaderen. This work is supported by the Deutsche Forschungsgemeinschaft within the wetting priority programme under grant Lo 418/5-3.

\section{References}

[1] Pandit R and Fisher M E 1983 Phys. Rev. Lett. 511772

[2] Dietrich S 1988 Phase Transitions and Critical Phenomena vol 12, ed C Domb and J L Lebowitz (London: Academic) pp 1-128

[3] Courtemanche D J and van Swol F 1992 Phys. Rev. Lett. 692078

[4] Dijkstra M 2004 Phys. Rev. Lett. 93108303

[5] Ohnesorge R, Löwen H and Wagner H 1994 Phys. Rev. E 504801

[6] Auer S and Frenkel D 2003 Phys. Rev. Lett. 91015703

[7] Löwen H 2001 J. Phys.: Condens. Matter 13 R415

[8] Bechinger C 2002 Curr. Opin. Colloid Interface Sci. 7204

[9] Choudhury A, Ackerson B J and Clark N A 1985 Phys. Rev. Lett. 55833

[10] Wei Q, Bechinger C, Rudhardt D and Leiderer P 1998 Phys. Rev. Lett. 812606

[11] Loudiyi K and Ackerson B J 1992 Physica A 18426 Chakrabarti J, Krishnamurthy H R, Sood A K and Sengupta S 1995 Phys. Rev. Lett. 752232

[12] Barrat J L and Xu H 1990 J. Phys.: Condens. Matter 29445 Chakrabarti J, Krishnamurthy H R and Sood A K 1994 Phys. Rev. Lett. 732923

[13] Radzihovsky L, Frey E and Nelson D R 2001 Phys. Rev. E 63031503

[14] Diestler D J, Schoen M, Curry J E and Cushman J H 1994 J. Chem. Phys. 1009140

[15] Schoen M 1996 J. Chem. Phys. 1052910

[16] Schmidt M and Löwen H 1997 Phys. Rev. E 557228

[17] Messina R and Löwen H 2003 Phys. Rev. Lett. 91146101

[18] Schoen M and Diestler D J 1997 Phys. Rev. E 564427

[19] Frink L J D and Salinger A G 1999 J. Chem. Phys. 1105969

[20] Bock H and Schoen M 1999 Phys. Rev. E 594122

[21] Bauer C, Dietrich S and Parry A O 1999 Europhys. Lett. 47474

[22] Bauer C and Dietrich S 1999 Phys. Rev. 606919

[23] A review is given in Xia Y, Qin D and Yin Y 2001 Curr. Opin. Colloid Interface Sci. 654

[24] See, e.g. Friebel S, Aizenberg J, Abad S and Wiltzius P 2000 Appl. Phys. Lett. 772406

[25] Burmeister F, Badowsky W, Braun T, Wieprich S, Boneberg J and Leiderer P 1999 Appl. Surf. Sci. 145461

[26] Burmeister F, Schäfle C, Keilhofer B, Bechinger C, Boneberg J and Leiderer P 1998 Chem. Eng. Technol. 21761

[27] Mio C and Marr D W M 1999 Langmuir 158565

[28] Aizenberg J, Braun P V and Wiltzius P 2000 Phys. Rev. Lett. 842997

[29] Chen K M, Jiang X, Kimerling L C and Hammond P T 2000 Langmuir 167825

[30] Murray C A 2000 Abstr. Pap. Am. Chem. 22074

[31] Mangold K, Bubeck R, Leiderer P and Bechinger C 2001 Prog. Colloid Polym. Sci. 11877

[32] Adamczyk Z, Siwek B and Musial E 2001 Langmuir 174529

[33] Guo Q, Arnoux C and Palmer R E 2001 Langmuir 177150

[34] Badilescu S, Truong V-V, Rochon P and Natansohn A 2001 Appl. Phys. Lett. 79872

[35] Heni M and Löwen H 2000 Phys. Rev. Lett. 853668

[36] Heni M and Löwen H 2001 J. Phys.: Condens. Matter 134675

[37] Löwen H, Allahyarov E, Dzubiella J, von Ferber C, Jusufi A, Likos C N and Heni M 2001 Phil. Trans. R. Soc. A 359909

[38] Hoogenboom J P, Vergeer P and van Blaaderen A 2003 J. Chem. Phys. 1193371

[39] van Blaaderen A, Ruel R and Wiltzius P 1997 Nature 385321 
[40] van Blaaderen A 1997 Prog. Colloid Polym. Sci. 10459

[41] van Blaaderen A and Wiltzius P 1997 Adv. Mater. 9833

[42] Arora A K and Rajagopalan R 1997 Curr. Opin. Colloid Interface Sci. 2391

[43] Hsu J-P and Tseng M-T 1997 J. Colloid Interface Sci. 188193

[44] Hoogenboom J P, van Langen-Suurling A K, Romijn J and van Blaaderen A 2003 Phys. Rev. Lett. 90138301

[45] Velikov K P, Christova C G, Dullens R P A and van Blaaderen A 2002 Science 296106

[46] Heni M and Löwen H 1999 Phys. Rev. E 607057

[47] Davidchack R L and Laird B B 2000 Phys. Rev. Lett. 854751

[48] Cacciuto A, Auer S and Frenkel D 2003 J. Chem. Phys. 1197467

[49] Heni M and Löwen H 2002 Phys. Rev. E 65021501

[50] Reichert H, Klein O, Dosch H, Denk M, Honkimäki V, Lippmann T and Reiter G 2000 Nature 408839

[51] Harreis H M, Schmidt M and Löwen H 2002 Phys. Rev. E 65041602

[52] Lin K H et al 2000 Phys. Rev. Lett. 851770

[53] Seguin J L et al 1983 Phys. Rev. Lett. 51122 Bienfait M et al 1984 Phys. Rev. B 29983

Krim J et al 1984 Phys. Rev. Lett. 52640

[54] Mistura G et al 1999 Phys. Rev. Lett. 82795

Bruschi L and Mistura G 2000 Phys. Rev. B 614941

Bruschi L and Mistura G 2001 J. Chem. Phys. 1141350

[55] Qiao Y and Christenson H K 1999 Phys. Rev. Lett. 831371

[56] Klier J, Weichhard C and Leiderer P 2000 Physica B 284391

[57] Gittes F T and Schick M 1984 Phys. Rev. B 30209

[58] Esztermann A, Heni M, Löwen H, Klier J, Sohaili M and Leiderer P 2002 Phys. Rev. Lett. 88055702

[59] Dietrich S and Schick M 1986 Phys. Rev. B 334952

[60] Pengra D B, Zhu D M and Dash J G 1991 Surf. Sci. 245125

[61] Landau L D and Lifshitz E M 1986 Theory of Elasticity 3rd edn (Oxford: Pergamon)

[62] $C$ is from Cheng E et al 1993 Phys. Rev. B 4818214 for $\mathrm{H}_{2}$ on $\mathrm{Ag}, \mathrm{Au}$, and $\mathrm{Cu}$; $E$ and $v$ are from Quesnel D J et al 1993 Phys. Rev. B 486795 $\rho_{\mathrm{s}}$ and $\rho_{\mathrm{g}}$ are from Agrawal R and Kofke D A 1995 Mol. Phys. 8543

[63] Hoogenboom J P, Yethiraj A, van Langen-Suurling A K, Romijn J and van Blaaderen A 2002 Phys. Rev. Lett. 89256104

[64] Allahyarov E, D’Amico I and Löwen H 1999 Phys. Rev. E 603199

[65] Hansen J-P and Löwen H 2000 Annu. Rev. Phys. Chem. 51209

[66] Lobaskin V, Lyubartsev A and Linse P 2001 Phys. Rev. E 63020401

[67] Terao T and Nakayama T 2002 Phys. Rev. E 65021405

[68] Biben T and Hansen J-P 1994 J. Phys.: Condens. Matter 6 A345

[69] Simonin J-P 1995 J. Phys. Chem. 991577

[70] Löwen H 1998 J. Phys.: Condens. Matter 10 L479

[71] Tellez G and Biben T 2000 Eur. Phys. J. E 2137

[72] van Roij R 2003 J. Phys.: Condens. Matter 15 S3569

[73] Esztermann A and Löwen H 2004 Europhys. Lett. 68120 